\title{
Author Correction: Isolated terawatt attosecond hard X-ray pulse generated from single current spike
}

Chi Hyun Shim ${ }^{1,2}$, Yong Woon Parc ${ }^{2}$, Sandeep Kumar ${ }^{3}$, In Soo Koํㄹ \&ong Eon Kim ${ }^{4,5}$

Correction to: Scientific Reports https://doi.org/10.1038/s41598-018-25778-x, published online 10 May 2018

This Article contains an error in Figure 4 where the key is incorrect in panels (d), (e), and (f). The correct Figure 4 appears below as Figure 1.
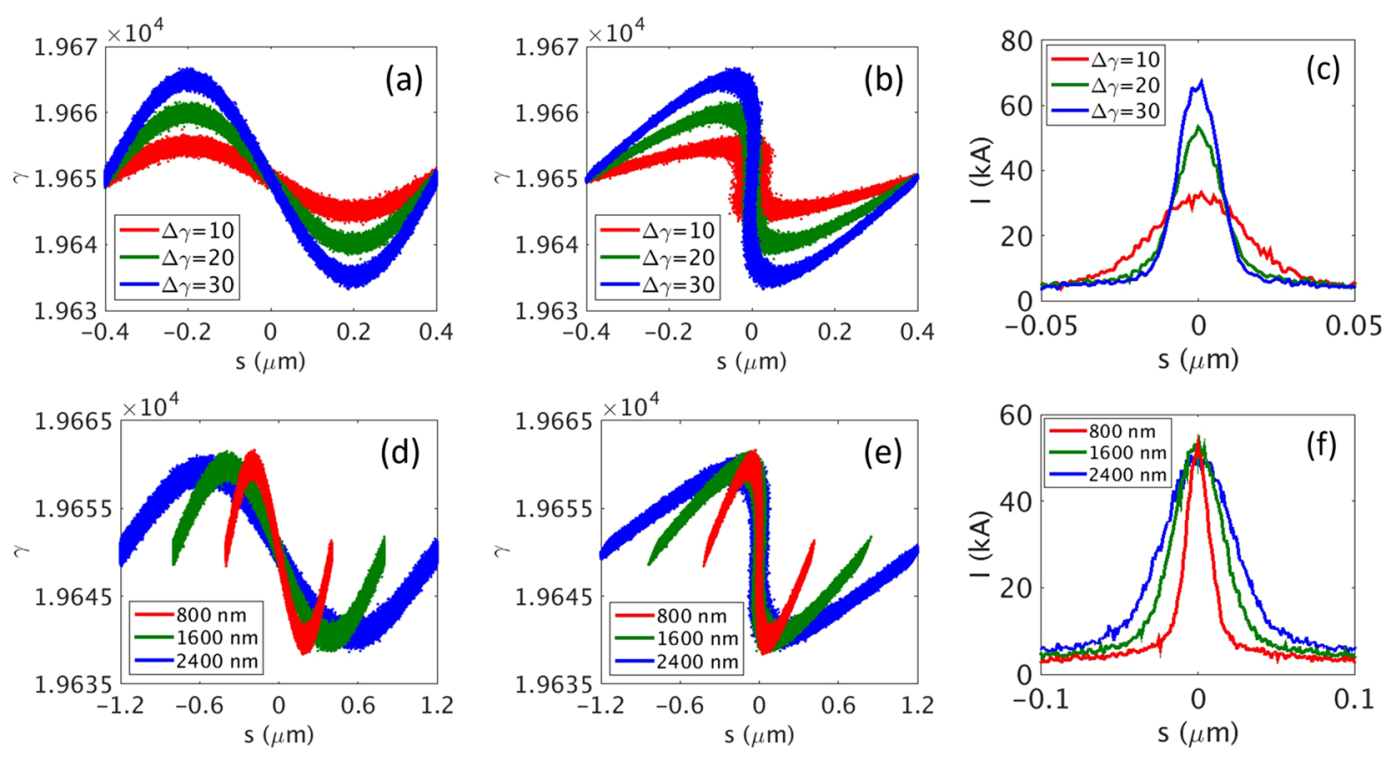

Figure 1. Effect of the energy and the wavelength of a modulation laser on the current spike formation. (a-c) Higher laser energy leads to higher energy modulation in the electron beam. Here, we examine the effect of the energy of a modulation laser in view of different values of energy modulation $(\Delta \gamma)$ in the electron beam: $\Delta \gamma=10$ (in red color), 20 (in green color), and 30 (in blue color) at a wavelength of $800 \mathrm{~nm}$ for the modulation laser. (d-f) Effect of the wavelength of the modulation laser: $800 \mathrm{~nm}$ (in red color), $1600 \mathrm{~nm}$ (in green color), and $2400 \mathrm{~nm}$ (in blue color) for $\Delta \gamma=20$. (a,d) Energy of the electron beam is modulated after a wiggler. (b,e) The distribution is distorted in the phase space after a chicane. (c,f) Current profile of the electron beam after chicane, showing a current spike.

${ }^{1}$ Department of Physics, Pohang University of Science and Technology, Pohang, 37673, Korea. ${ }^{2}$ Pohang Accelerator Laboratory, Pohang University of Science and Technology, Pohang, 37673, Korea. ${ }^{3}$ Department of Physics, Ulsan National Institute of Science and Technology, Ulsan, 44919, Korea. ${ }^{4}$ Department of Physics, Center for Attosecond Science and Technology, Pohang University of Science and Technology, Pohang, 37673, Korea. ${ }^{5}$ Max Planck POSTECH/Korea Res. Init., Pohang, 37673, Korea. Correspondence and requests for materials should be addressed toY.W.P. (email: young1@postech.ac.kr) or D.E.K. (email: kimd@postech.ac.kr) 
(i) Open Access This article is licensed under a Creative Commons Attribution 4.0 International License, which permits use, sharing, adaptation, distribution and reproduction in any medium or format, as long as you give appropriate credit to the original author(s) and the source, provide a link to the Creative Commons license, and indicate if changes were made. The images or other third party material in this article are included in the article's Creative Commons license, unless indicated otherwise in a credit line to the material. If material is not included in the article's Creative Commons license and your intended use is not permitted by statutory regulation or exceeds the permitted use, you will need to obtain permission directly from the copyright holder. To view a copy of this license, visit http://creativecommons.org/licenses/by/4.0/.

(C) The Author(s) 2019 